\title{
Excretory-Secretory Products from Hookworm
}

\section{$L_{3}$ and Adult Worms Suppress Proinflammatory Cytokines in Infected Individuals}

\author{
Stefan Michael Geiger, ${ }^{1}$ Ricardo Toshio Fujiwara, ${ }^{1,2}$ Paula Albuquerque Freitas, ${ }^{1}$ \\ Cristiano Lara Massara, ${ }^{1}$ Omar dos Santos Carvalho, ${ }^{1}$ Rodrigo Corrêa-Oliveira, ${ }^{1}$ \\ and Jeffrey Michael Bethony ${ }^{1,3}$ \\ ${ }^{1}$ Centro de Pesquisas René Rachou, Fundação Oswaldo Cruz, Avenida Augusto de Lima 1715, 30190-002 \\ Belo Horizonte, MG, Brazil \\ ${ }^{2}$ Departamento de Parasitologia, Instituto de Ciências Biológicas, Universidade Federal de Minas Gerais, Avenida Antônio Carlos 6627, \\ 31270-901 Belo Horizonte, MG, Brazil \\ ${ }^{3}$ Department of Microbiology, Immunology, and Tropical Medicine, The George Washington University Medical Center, \\ 2300 Eye Street NW, Washington, DC 20037, USA
}

Correspondence should be addressed to Stefan Michael Geiger, stefan@cpqrr.fiocruz.br

Received 30 November 2010; Revised 18 March 2011; Accepted 29 March 2011

Academic Editor: Takeshi Agatsuma

Copyright (c) 2011 Stefan Michael Geiger et al. This is an open access article distributed under the Creative Commons Attribution License, which permits unrestricted use, distribution, and reproduction in any medium, provided the original work is properly cited.

\begin{abstract}
We compared the effects of larval and adult worm excretory-secretory (ES) products from hookworm on the proliferative responses and cytokine secretion in peripheral blood mononuclear cells (PBMCs) from hookwormpatients and egg-negative, nonendemic controls. When compared with negative controls, mitogen-stimulated PBMC from hookworm-infected individuals showed a significantly reduced proliferative response when adult worm ES antigen was added to the cultures. Furthermore, in hookworm-infected individuals a significant downmodulation of inflammatory interleukin (IL)-6 and tumor necrosis factor (TNF) $-\alpha$ secretion resulted when PBMCs were stimulated with mitogen in combination with larval or adult worm ES. Both, interferon (IFN) $-\gamma$ and IL-10 secretion were significantly lower in stimulated PBMC from infected individuals; however the IFN$\gamma / \mathrm{IL}-10$ ratio was much lower in hookworm-infected patients. Comparable effects, although at lower concentrations, were achieved when PBMCs from both groups were incubated with living hookworm third-stage larvae. We suggest that hookworm ES products downmodulate proliferative responses and inflammation during the chronic phase of the disease and facilitate early larval survival or adult worm persistence in the gut.
\end{abstract}

\section{Introduction}

Helminth excretory-secretory products (ES) contain a vast mixture of antigens, are potent modulators of the host's immune response, and therefore are important factors in worm survival and maintenance of a chronic human infection. ES products from different parasite species and different stages of parasitic development within the host were shown to downmodulate Type 1 immunity [1]. Among other mechanisms, early effects on dendritic cell function and innate immune responses have been previously described for intestinal nematode models [2], as well as for filarial in- fections $[3,4]$ with these events contributing to minimize inflammatory responses and induce a Type 2 response [1]. For hookworms, many components in ES products have been described so far $[5,6]$; however, their effect on the human immune response is still not well understood and has become, more recently, a topic of intense investigation.

We have recently reported that PBMCs from Necator americanus-infected patients had a lower production of TNF- $\alpha$ and IL-10 in response to ES antigen derived from adult Ancylostoma caninum. Apart from the downmodulated cytokine secretion in response to ES antigen, PBMCs from endemic patients proliferated poorly in response to crude 
ES antigen extracts [7]. In the present study, we compare the effects of hookworm adult and larval ES antigen preparations on polyclonal-activated lymphocytes from two different groups-(1) individuals chronically infected with hookworm, residing in an area of high transmission and (2) egg-negative ("control") and nonexposed individuals, residing in a nonendemic area. We found that proliferative responses and the secretion of proinflammatory cytokines in hookworm-infected individuals were downmodulated to a significant degree when compared to egg-negative controls.

\section{Materials and Methods}

2.1. Selection of Patients. In the present study, hookworminfected adult individuals were recruited during an epidemiological field survey in 2006 in the village of Ladainha, located in the north-eastern region of Minas Gerais state, Brazil. A single stool sample was collected from individuals and eggs per gram of feces (epg) determined by the Kato-Katz fecal thick-smear technique [8] with two slides per patient. Individuals found to be monoinfected with hookworm were then enrolled into this study $(n=10)$. Blood was taken by venipuncture and the patients subsequently treated with a single dose of albendazole $(400 \mathrm{mg})$ by the local health service personnel. Approximately $20 \mathrm{~mL}$ of blood was collected in heparinized tubes for separation of peripheral blood mononuclear cells (PBMCs). Nonendemic, eggnegative controls $(n=7)$ were recruited from the urban area of Belo Horizonte, Minas Gerais, which is considered an area of low to negligible transmission, especially outside the poor urban areas. Egg-negative controls consisted of volunteers from Centro de Pesquisas René Rachou who have not reported any intestinal helminth infection in the past. All volunteers provided written informed consent to participate in the study, and it was approved by the ethics committee from Centro de Pesquisas René Rachou-FIOCRUZ and the Brazilian "Conselho Nacional de Ética em Pesquisa" (CONEP).

\subsection{Parasite ES Antigen Preparation. Ancylostoma caninum} adult worms were recovered from the small intestines of stray dogs euthanized at the dog kennel of the Prefecture of Belo Horizonte during the regular leishmaniasis control program. Adult worm ES products (ES-AW) were obtained after incubation of the parasites for a period of 16-20 hours at $37^{\circ} \mathrm{C}$ in a humidified incubator and stored in aliquots at $-70^{\circ} \mathrm{C}[9]$. ES preparations from several days were pooled into $15 \mathrm{~mL}$ filtration tubes with a $5 \mathrm{kDa}$ molecular weight cut-off filter (Millipore) and centrifuged for 1 hour at $4^{\circ} \mathrm{C}$ and $1,250 \mathrm{~g}$. Antigen preparations were obtained after filtration in a $0.22 \mu \mathrm{m}$ low-protein binding syringe filter (Millipore) and the resulting protein concentration determined using the BCA protein assay kit (Pierce).

In order to obtain $\mathrm{L}_{3}$ for preparation of ES products, Harada-Mori fecal cultures from hookworm-infected individuals with more than 4,000 epg were performed [10]. Fiftymili-liter plastic tubes was filled with $5 \mathrm{~mL}$ of tap water. Feces were distributed on filter paper strips on the upper two thirds of the filter paper, transferred to plastic tubes, and incubated in vertical position at $26-28^{\circ} \mathrm{C}$ for $7-10$ days. The tubes were sealed with perforated Parafilm for air circulation. Fecal cultures were checked daily for water level, fungal contamination, and larvae in the water. After 7-10 days of incubation, the liquid was pooled in new tubes. To separate $\mathrm{L}_{3}$ from fecal material and fungi the tubes were thoroughly mixed on a vortex and left on the bench during 30 minutes for sedimentation. After carefully removing the supernatant the content of the tubes were merged and the pellet with $\mathrm{L}_{3}$ was resuspended with $40 \mathrm{~mL}$ of BU buffer at room temperature, as described by Hawdon et al. [11]. The solution was transferred to a small Baerman filter unit, containing several layers of gauze, and incubated for one hour at room temperature (RT). The resulting pellet of larvae was resuspended with BU buffer, mixed thoroughly, and the sedimentation step repeated. For bacterial decontamination, the pellet was incubated in $\mathrm{BU} / 1 \% \mathrm{HCl}$ buffer for 30 minutes at RT [11]. The larval suspension was transferred to a sterile $50 \mathrm{~mL}$ plastic tube and resuspension and sedimentation steps repeated under sterile conditions once with BU buffer, twice with minimal essential medium (Gibco), and once with RPMI-1640 medium (Gibco), containing 10\% heatinactivated normal human serum (ICN), $2 \%$ antibioticantimycotic solution (Sigma), and 1\% L-glutamine (Winlab, Leicestershire, UK). After the last sedimentation step, the supernatant was removed, and the larvae resuspended in $5 \mathrm{~mL}$ complete RPMI-1640 medium (see above). For activation of larvae, $1 \mathrm{~mL}$ of larval suspension, containing approximately 10,000-20,000 larvae, was incubated in $3 \mathrm{~mL}$ of complete RPMI-1640 medium in 8-well cell multidish plates (NUNC) for $16-18$ hours at $37^{\circ} \mathrm{C}$ and $5 \% \mathrm{CO}_{2}$. Finally, the larval suspension was removed, pooled into $1.5 \mathrm{~mL}$ cups, and centrifuged at 20,800 g for 3 minutes. The supernatant was carefully removed and antigen preparations (ES- $\mathrm{L}_{3}$ ) from different larval cultures were pooled and stored at $-70^{\circ} \mathrm{C}$ until used. Due to serum supplementation of cell culture medium for activation of $\mathrm{L}_{3}$, it was not possible to determine the specific protein concentration of larval ES products.

\subsection{In Vitro Lymphocyte Proliferation. Separation of PBMC} was performed as described elsewhere [12]. In 96-well cell culture plates, triplicates of 250,000 cells/well were added for antigen and mitogen stimulations in a final volume of $200 \mu \mathrm{L}$ of complete RPMI-1640 [12]. Final concentrations of stimulants determined to be optimal in cell culture were $35 \mu \mathrm{g} / \mathrm{mL}$ for ES-AW antigens and $2.5 \mu \mathrm{g} / \mathrm{mL}$ for phytohaemagglutinin (PHA)-L (Difco Laboratories, Detroit, MI, USA). For proliferation assays with ES- $\mathrm{L}_{3}$ antigen, a dose-response curve was performed at the beginning of the experiments, in which an increasing volume of $\mathrm{L}_{3}$ supernatant $(5-50 \mu \mathrm{L})$ was added to mitogen-stimulated PBMCs. $25 \mu \mathrm{L}$ of $\mathrm{L}_{3}$ supernatant resulted in a $56 \%$ inhibition of PBMC proliferation (data not shown) and was used in all subsequent experiments of proliferation and cytokine secretion. Cells were cultured at $37^{\circ} \mathrm{C}$ in a humidified $5 \% \mathrm{CO}_{2}$ incubator. Tritiated thymidine (Amersham Pharmacia, São Paulo, Brazil; $0.5 \mu \mathrm{Ci} /$ culture; 
specific activity $6.7 \mathrm{Ci} / \mathrm{mM}$ ) was added to the cultures at $48 \mathrm{~h}$, and the cells were harvested $18 \mathrm{~h}$ later. Incorporated tritiated thymidine was determined in a liquid scintillation counter and the data expressed as stimulation indices (SIs) (mean proliferation of stimulated culture divided by mean proliferation of unstimulated culture). The stimulation index of PHA-stimulated lymphocytes served as reference value (100\%), and percentages of costimulated cell cultures, either PHA plus ES-AW or PHA plus ES- $\mathrm{L}_{3}$, were calculated from this value.

2.4. Cytokine Detection in Cell Culture Supernatants. For production of cytokines and chemokines, $5 \times 10^{5}$ PBMCs were cultivated in 48-well tissue culture plates (Costar, Corning, NY, USA) at a total volume of $400 \mu \mathrm{L}$ in complete RPMI-1640 for 2 days, using the same final mitogen and antigen concentrations as described above. Cell-free supernatants were stored at $-70^{\circ} \mathrm{C}$ until cytokine quantification. Concentrations for IL- $1 \beta$, IL-5, IL-6, IL-10, IFN- $\gamma$, and TNF- $\alpha$ were determined by enzyme-linked immunosorbent assay (ELISA) (R\&D Systems, Minneapolis, USA). When necessary, samples were diluted with PBS in order to obtain a value within the range of the standard curve. ELISAs were performed in duplicate according to the manufacturer's protocols, using a total volume of $25 \mu \mathrm{L}$ per well in highbinding half-area plates (COSTAR, Corning, NY, USA). On each plate, serial dilutions of standards were run to construct standard curves with the following ranges of concentration: IL-1 $\beta$ (3.9-500 pg/mL); IL-5 (11.7-1,500 pg/mL); IL$6(4.7-600 \mathrm{pg} / \mathrm{mL}) ; \mathrm{IL}-10(23.4-3,000 \mathrm{pg} / \mathrm{mL}) ; \mathrm{IFN}-\gamma(7.8-$ $1,000 \mathrm{pg} / \mathrm{mL}) ;$ TNF- $\alpha(7.8-1,000 \mathrm{pg} / \mathrm{mL})$. The sensitivity for all ELISAs was lower than the last standard dilution. The colorimetric reaction was determined in an automated ELISA reader at $450 \mathrm{~nm}$. Back calculations of cytokine concentrations from mean optical density values were interpolated from the standard curves by using a 4-parameter curve fitting program (SOFTmax PRO 3.1.2).

2.5. Cultivation of Lymphocytes and Living $L_{3}$. For co-cultivation of PBMC with infective hookworm larvae, $\mathrm{L}_{3}$ from Ancylostoma caninum-infected dogs were obtained after fecal cultures (see above) and were a kind donation of Professor Walter dos Santos Lima and Professor Joziana Barçante (Federal University of Minas Gerais-UFMG). The in vitro cell cultures, $5 \times 10^{5} \mathrm{PBMCs}$, were cultivated in 24-Transwell plates (pore size $3.0 \mu \mathrm{m}$, COSTAR, Corning, NY, USA), at a total volume of $400 \mu \mathrm{L}$ in complete RPMI-1640. Cells were incubated, either in direct contact or separated by the plate insert, with approximately $20 \mathrm{~L}_{3}$ per well during 48 hours. Cell supernatants were obtained as described above and were stored at $-70^{\circ} \mathrm{C}$ for future cytokine determination. Cytokine ELISAs were performed as described above.

2.6. Statistical Methods. For statistical evaluation, an SPSS 12.0 software package was used. Values from proliferation assays and cytokine concentrations were checked for normal distribution and were subsequently analysed by the nonparametric Mann-Whitney $U$-test for the comparison of two

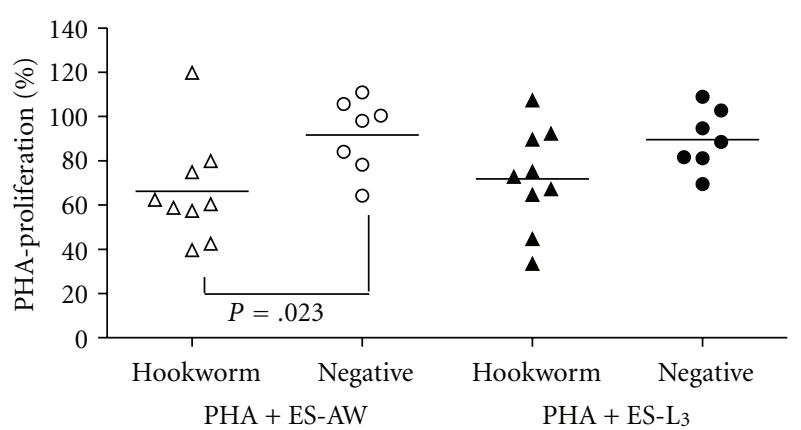

FIGURE 1: Inhibition of mitogen-stimulated PBMC proliferation after costimulation with adult worm ES (ES-AW, open symbols) and third-stage larval ES (ES- $\mathrm{L}_{3}$, full symbols) antigen in hookworminfected $(\boldsymbol{\Delta} ; n=9)$ and egg-negative $(\bullet ; n=7)$ individuals. Symbols show individual percentages of proliferation in comparison with PHA-stimulated cultures only, and horizontal bars indicate mean values for each group. Significant differences between groups are indicated.

groups. Differences with a $P$ value of less than .05 were considered significant and are indicated in the text, table, or figure.

\section{Results}

3.1. Patients and Parasitological Exams. The mean age of hookworm-infected patients from Ladainha was higher than that in negative, nonendemic controls from Belo Horizonte $(44.4 \pm 13.8$ years versus $29.4 \pm 5.8$ years $)$; however this difference was not significant $(P=.055)$. Also, nonendemic controls have been living in the urban area of Belo Horizonte. For the egg-negative group, helminth infections were not diagnosed at the time of the study and no past records of helminth infections were stated by the volunteers from the hypoendemic area. The geometric mean of hookworm egg counts was $789 \mathrm{epg}$ (range: 11,892-684), with 7 patients harbouring light, 2 moderate, and 1 patient with heavy hookworm infections, according to the classification by the World Health Organization [13].

3.2. Lymphocyte Proliferation. Figure 1 shows the individual percentages of PBMC proliferation after costimulation with PHA and hookworm ES-AW or ES- $\mathrm{L}_{3}$ in comparison to PHA-stimulated lymphocytes only. PBMCs from hookworm patients showed a lower percentage of proliferation when hookworm ES antigens were added to the lymphocyte cultures. After addition of ES-AW to PHA-stimulated lymphocytes the percentage of proliferation was significantly lower $(P=.023)$ in hookworm-infected individuals. Notably, when PBMC cultures were stimulated with PHA only, no significant differences in the stimulation index resulted between the two patient groups (data not shown).

3.3. Cytokine Secretion. Cytokine concentrations detected in cell culture supernatants are shown in Table 1.

In unstimulated PBMC cultures from negative, nonendemic individuals lower concentrations of inflammatory IL-1 $\beta$, IL-6, and TNF- $\alpha$ were detected when compared 
TABLE 1: Cytokine concentrations $(\mathrm{pg} / \mathrm{mL})$ in supernatants from PHA-stimulated PBMC cultures with or without costimulation by adult worm ES (ES-AW) or third-stage larval ES $\left(E S-\mathrm{L}_{3}\right)$ antigen. Values from hookworm-infected individuals $(n=7)$ and negative controls $(n=6)$ are compared and indicated as mean values \pm standard errors. Significant differences between groups are indicated with an asterisk $\left({ }^{*} P<.01 ;{ }^{* *} P<.05\right)$.

\begin{tabular}{lccccc}
\hline & Group & Control & PHA & PHA+ES-AW & PHA+ES-L 3 \\
\hline IL-1 $\beta$ & Hookworm & $1,034 \pm 239^{*}$ & $1,446 \pm 176^{*}$ & $1,421 \pm 265$ & $1,456 \pm 320$ \\
& Negative controls & $27 \pm 27^{*}$ & $215 \pm 114^{*}$ & $1,562 \pm 424$ & $785 \pm 340$ \\
IL-6 & Hookworm & $52,382 \pm 14,323$ & $26,553 \pm 12,000^{* *}$ & $27,865 \pm 7,464^{*}$ & $22,056 \pm 4,763^{*}$ \\
& Negative controls & $18,452 \pm 4,400$ & $63,409 \pm 14,530^{* *}$ & $102,499 \pm 11,146^{*}$ & $87,143 \pm 14,244^{*}$ \\
TNF- $\alpha$ & Hookworm & $3,630 \pm 2,621^{* *}$ & $297 \pm 185^{* *}$ & $741 \pm 300^{*}$ & $885 \pm 333^{* *}$ \\
& Negative controls & $306 \pm 114^{* *}$ & $2,104 \pm 538^{* *}$ & $5,638 \pm 1,385^{*}$ & $2,820 \pm 832^{* *}$ \\
IL-5 & Hookworm & $35 \pm 19^{* *}$ & $40 \pm 20$ & $12 \pm 7$ & $5 \pm \pm 40$ \\
& Negative controls & $5 \pm 5^{* *}$ & $45 \pm 22$ & $225 \pm 69^{* *}$ & $37 \pm 17$ \\
IL-10 & Hookworm & $63 \pm 26$ & $113 \pm 48$ & $526 \pm 58^{* *}$ & $310 \pm 120^{* *}$ \\
& Negative controls & 0 & $157 \pm 33$ & $230 \pm 165^{*}$ & $376 \pm 89^{* *}$ \\
IFN- $\gamma$ & Hookworm & $43 \pm 22$ & $3,288 \pm 1,046^{*}$ & $370 \pm 899^{*}$ & $158 \pm 99^{*}$ \\
& Negative controls & 0 & & $2,168 \pm 796^{*}$ \\
\hline
\end{tabular}

with participants with hookworm infection. For IL-1 $\beta$ and TNF- $\alpha$, these differences were statistically significant $(P<.01$ and $P<.05$, resp. $)$. When stimulated with PHA, PBMC from hookworm-infected individuals secreted significantly more IL- $1 \beta(P<.01)$ than egg-negative control subjects. Opposite to unstimulated control cultures, IL-6 and TNF- $\alpha$ secretions in PHA-stimulated or costimulated cultures were significantly lower in PBMCs from hookworm patients than in egg-negative individuals. For IL-5, low concentrations were detected in both, control and stimulated lymphocyte cultures from the two groups. However, unstimulated PBMCs from hookworm patients secreted significantly more IL-5 $(P<.05)$ than PBMCs from eggnegative individuals. After addition of either ES-AW or ES$\mathrm{L}_{3}$ antigen to PHA-stimulated cell cultures, individuals in both groups showed increased IL-10 secretion. However, this increase was significantly $(P<.05)$ higher in the egg-negative controls. The IFN- $\gamma$ secretion in participants with hookworm infection was significantly $(P<.01)$ lower in all stimulated lymphocyte cultures when compared with egg-negative individuals. Figure 2 shows the paired IFN- $\gamma$ and IL-10 secretion for the two groups of patients separated in egg-negative controls (2A) and hookworminfected individuals (2B). Upon stimulation, PBMC from egg-negative individuals secreted high levels of IFN- $\gamma$ and considerably lower levels of IL-10. In contrast, PBMC from hookworm patients secreted equally low concentrations of IFN- $\gamma$ and IL-10 (Figures 2(a) and 2(b)).

3.4. Cultivation of Lymphocytes and Living $L_{3}$. Table 2 shows the results obtained for cytokine secretion levels from PBMC cocultured with living $\mathrm{L}_{3}$ of $A$. caninum. Although the concentrations are lower if compared with the previous data, the differences in the secretion of inflammatory cytokines (IL-6, TNF- $\alpha$ ) after co-cultivation with living $\mathrm{L}_{3}$ showed the same trend as described for soluble ES-L $\mathrm{L}_{3}$ preparations from $N$. americanus (Table 1 ). These results show that this regulatory effect may be mediated by soluble factors secreted

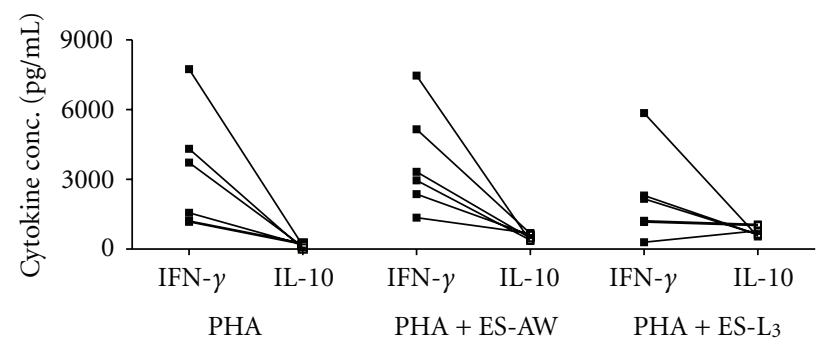

(a)
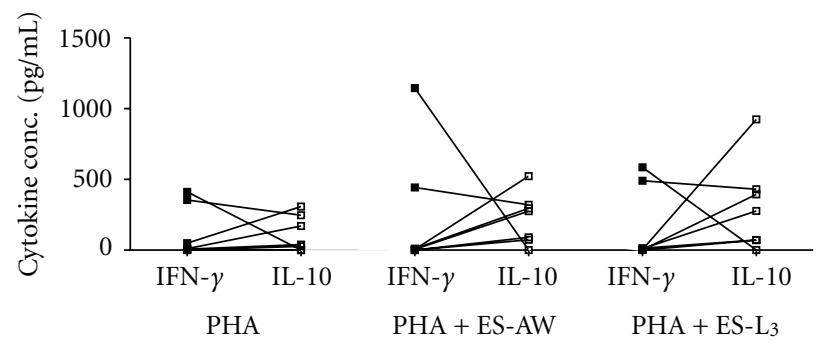

(b)

FIGURE 2: IFN- $\gamma$ and IL-10 secretion in $\mathrm{pg} / \mathrm{mL}$ in PBMC stimulated with PHA, PHA+ adult worm ES (ES-AW), or PHA+ ES-L L $_{3}$ (a) Paired IFN- $\gamma$ and IL-10 cytokine concentrations in PBMCs from egg negative, nonendemic controls and (b) in hookworm-infected patients from an endemic area.

by the $\mathrm{L}_{3}$. On the other hand and in contrast with the soluble antigen preparations, co-cultivation of $\mathrm{PBMC}$ and $\mathrm{L}_{3}$ did not induce an increase in IL-10 secretion and cytokine concentrations remained low with no significant differences between the two groups (Table 2). IL-5 and IFN- $\gamma$ secretion in PBMC cocultured with living $\mathrm{L}_{3}$ remained at marginal levels in both patient groups (data not shown).

\section{Discussion}

Suppressed cellular responsiveness, either antigen-specific or polyclonal, and a skewed Th2 immune response are two of 
TABLE 2: Cytokine concentrations ( $\mathrm{pg} / \mathrm{mL})$ in supernatants of PBMC cultures cocultured with living $\mathrm{L}_{3}$ from Ancylostoma caninum. Cells were either separated from the $\mathrm{L}_{3}$ by a membrane insert $\left(\right.$ cells $\left./ \mathrm{L}_{3}\right)$ or were cultivated in direct contact with the parasites $\left(\right.$ cells $\left.+\mathrm{L}_{3}\right)$. Values from hookworm-infected individuals $(n=7)$ and negative controls $(n=6)$ are compared and indicated as mean values \pm standard errors. Significant differences between patient groups are indicated with an asterisk $\left({ }^{*} P<.01 ; * * P<.05\right)$.

\begin{tabular}{lcccc}
\hline & Group & Control & Cells/L $\mathrm{L}_{3}$ & Cells $+\mathrm{L}_{3}$ \\
\hline IL-1 $\beta$ & Hookworm & $1,034 \pm 239^{*}$ & $833 \pm 149$ & $1,161 \pm 218$ \\
& Negative controls & $27 \pm 27^{*}$ & $424 \pm 134$ & $1,470 \pm 494$ \\
IL-6 & Hookworm & $52,382 \pm 14,323$ & $8,703 \pm 2,258^{*}$ & $12,124 \pm 2,548^{*}$ \\
& Negative controls & $18,452 \pm 4,400$ & $62,750 \pm 18,488^{*}$ & $59,185 \pm 15,443^{*}$ \\
TNF- $\alpha$ & Hookworm & $3,630 \pm 2,621^{* *}$ & $351 \pm 110^{*}$ & $404 \pm 94^{*}$ \\
& Negative controls & $306 \pm 114^{* *}$ & $1,784 \pm 362^{*}$ & $2,163 \pm 221^{*}$ \\
IL-10 & Hookworm & $63 \pm 26$ & $86 \pm 48$ & $37 \pm 26$ \\
& Negative controls & 0 & $18 \pm 16$ & $92 \pm 31$ \\
\hline
\end{tabular}

the hallmarks of helminth infections and have been extensively described for filarial infections $[14,15]$ and schistosomes [16-18]. In two independent studies, we have found reduced antigen-specific cellular responsiveness in hookworm patients in response to larval and adult worm soluble extracts $[7,19]$. Interestingly, proliferative responses and cytokine secretion patterns were quite distinct for the different antigen preparations, with adult ES antigen inducing low type 1 and inflammatory responses especially in hookworm-infected individuals [7].

In the present paper, we have focused on the cellular response to ES antigenic preparations from two developmental stages of hookworms-infective third-stage larvae $\left(\mathrm{L}_{3}\right)$ and adult worms (AWs). We were able to show that both ES preparations, $\mathrm{L}_{3}$ and $\mathrm{AW}$, induce a considerable reduction in cell proliferative responses of polyclonally stimulated lymphocytes from hookworm-infected patients. The fact that we observe a reduction on polyclonal activation of PBMCs in infected participants with $\mathrm{L}_{3}$ ES antigen, suggests that down-modulatory mechanisms occur at the early $\mathrm{L}_{3}$ parasitic stage of infection in individuals previously sensitized with parasite antigens or that have a current infection. This observation is novel for hookworm infections and is certainly of major importance for vaccine development. In this context, induction of regulatory/suppressor responses induced by ES $\mathrm{L}_{3}$ antigens may have significant effect on the maintenance of parasite survival as well as on reinfection in endemic human populations. Importantly, Loukas et al. [20] showed that $\mathrm{L}_{3}$ and adult worms from A. caninum share ES and somatic antigens; these results together with the results presented in this paper suggest that common mechanisms of $\mathrm{L}_{3}$ and adult worms on the regulation of the immune response may facilitate parasite escape and survival. In contrast, it has also been shown by others that mechanisms of immunomodulation and the induction of pathological changes cannot be generalised between different hookworm species and that the induced inflammatory response in humans infected with $N$. americanus is much more subtle than, for example, after enteric A. caninum infections [21]. Also, in primary experimental infections in human volunteers and in hamsters it was shown that a state of reduced cellular responsiveness builds up slowly during the onset of patency, most probably with the more important contribution of the adult worm population that builds up with time $[22,23]$.

Due to the obvious difficulties in obtaining sufficient ES material from living human hookworm species, we used in our experiments adult worm ES products, as well as living larvae from A. caninum and compared the human cellular response to that of $\mathrm{L}_{3} \mathrm{ES}$ products from $N$. americanus. We are well aware of the differences between human and dog hookworm infections [24] and that ES products from adult A. caninum, and $N$. americanus have been shown to have individual protein patterns and bind to distinct leukocyte populations [25] and different components might therefore have distinct effects on the human immune system [26]. However, the effects of total ES extracts, as described here, seem to be comparable for both dog and human hookworm ES product preparations. We are also aware of statistical limitations due to the low number of individuals in each group, which might impair to discover small differences between groups. However, we feel that in the present experimental setting with the unspecific polyclonal stimulation of lymphocytes by the mitogen PHA we are able to draw the presented conclusions, even if limited quantities of ES antigens forced us to use blood from a restricted number of individuals.

Experimental human infections monitored by capsule endoscopy have shown that adult worms still cause a significant degree of intestinal inflammation and newly arriving premature worms are expelled from the small intestine in the course of acute eosinophilic enteritis [27]. Nevertheless, adult hookworms have also been shown to resist intestinal inflammation and continued to parasitize the small intestine [28]. Furthermore, in repeatedly administered experimental human infections, even with as much as $250 \mathrm{~L}_{3}$, initial intestinal symptoms seem to vanish with every newly applied infection [29]. In our view, this indicates time- and dosedependent mechanisms of hookworm immunosuppression with decreased intestinal inflammation, resulting in parasite persistence. There are already described mechanisms by which adult worm ES components induce suppression of the human immune response, for example, by the induction of apoptosis in reactive $\mathrm{T}$ cells, which avoids the infiltration 
of reactive host leukocytes to the place of adult worm attachment and facilitates worm survival [30], or by affecting dendritic cell maturation and differentiation of regulatory $\mathrm{T}$ cells [31].

We observed elevated levels of IL-1 $\beta$, IL-6, and TNF$\alpha$ concentrations in unstimulated PBMC cultures from infected individuals indicating that these volunteers have ongoing inflammatory processes. However, after in vitro PBMC stimulation infected patients showed a reduced capacity in secreting IL-6 and TNF- $\alpha$ either after PHA stimulation or in combination with ES antigen from both parasitic stages. Together with a low IFN- $\gamma$ secretion in individuals with hookworm infection, our results point to a major role of ES products for reduced inflammatory/type 1 immune responses in the course of human hookworm infection. On the other hand, low to absent IL-5 secretion, either in response to ES preparations or in response to living parasites, respectively, suggests that ES antigens are poor inducers of type 2 immunity. This was also confirmed by previous antigen-specific stimulations of PBMC from hookworminfected subjects [7], and similar results were described in PBMCs from nonexposed individuals which were incubated with live $\mathrm{L}_{3}$ from the filarial parasite Brugia malayi [32]. As other mechanism influencing the immunoregulation and the cytokine and chemokine milieu, it was reported that host eotaxin is specifically cleaved by metalloproteases from adult $N$. americanus $\mathrm{ES}$ secretions, which might be important in preventing recruitment and activation of eosinophils and even may influence Th2 responses [33]. Therefore, it would be interesting in future studies to collect additional information on IL-4 or IL-13 lymphocyte secretion patterns, or even on IL-21, a Th2 cytokine identified to be highly relevant in helminth infections [34]. Furthermore, during schistosome infection and migration through the skin, IL10 seems to be a key regulator of the immune response [35]. However, in this study we observed a significantly lower IL-10 secretion in PBMC cultures from hookworm-infected patients when compared to negative individuals. On the other hand, a resulting immune response is the interplay between different and also counteracting cytokines. As such, the paired IFN- $\gamma$ and IL-10 secretion for each patient upon stimulation of PBMC with ES antigens showed a completely different pattern between the two patient groups; for example, upon stimulation there is a relatively higher IFN $-\gamma$ than IL-10 level in egg-negative controls and equally low levels of IFN- $\gamma$ and IL-10 in hookworm-infected individuals. In our view, this ES antigen-induced low IFN- $\gamma /$ IL-10 ratio in hookworm-infected patients may contribute to the enhanced suppression of the immune response, with reduced cellular reactivity, and a diminished inflammatory response. Interestingly, an elegant study in Heligmosomoides polygyrus-infected mice has recently shown that adult worm ES products from this rodent nematode inhibit the maturation and function of dendritic cells and may drive the differentiation of IL10 producing $\mathrm{T}_{\text {reg }}$ cells. As a result, $\mathrm{T}$ cell, cytokine, and antibody responses are suppressed in a generalized manner [36]. The putative role of an altered phenotype and function of dendritic cells in hookworm-infected patients on the observed suppression/regulation of the immune response in these patients has recently been published by our group [37]. Also, the important contribution of alternatively activated macrophages in the course of intestinal helminth infections has recently been reviewed and emphasized by Kreider et al. [38] and might be important in directing the immune response during human hookworm infections. As such, the effect of hookworm ES products on antigen presenting cells, as well as the induction of regulatory $\mathrm{T}$ cell cytokines, such as TGF- $\beta$ or IL-17, deserves further investigation.

In summary, we were able to show that hookworm ES products from A. caninum adult worms or $\mathrm{L}_{3}$ induce immune mechanisms that significantly reduce proliferative responses in mitogen-activated PBMC from hookworminfected individuals. Furthermore, a significant downmodulation of inflammatory cytokine secretion, as well as a lower IFN- $\gamma /$ IL-10 ratio, resulted in stimulated PBMC from hookworm patients, when compared to nonendemic, eggnegative controls, factors that might be decisive for early larval survival or adult worm persistence in the gut.

\section{Acknowledgments}

The authors are grateful to the patients from Ladainha and the nonendemic volunteers at Centro de Pesquisas René Rachou for their collaboration. Financial support for the study was provided by the J. Fogarty Foundation and by the PAPES V program from Fiocruz/CNPq. Also, They would like to thank Professor Walter dos Santos Lima and Professor Joziana Barçante (Federal University of Minas GeraisUFMG) for the donation of infective hookworm larvae. S. M. Geiger and R. T. Fujiwara contributed equally to this work.

\section{References}

[1] R. M. Maizels, A. Balic, N. Gomez-Escobar, M. Nair, M. D. Taylor, and J. E. Allen, "Helminth parasites-masters of regulation,” Immunological Reviews, vol. 201, pp. 89-116, 2004.

[2] A. Balic, Y. Harcus, M. J. Holland, and R. M. Maizels, "Selective maturation of dendritic cells by Nippostrongylus brasiliensissecreted proteins drives Th2 immune responses," European Journal of Immunology, vol. 34, no. 11, pp. 3047-3059, 2004.

[3] R. T. Semnani, M. Law, J. Kubofcik, and T. B. Nutman, "Filaria-induced immune evasion: suppression by the infective stage of Brugia malayi at the earliest host-parasite interface," Journal of Immunology, vol. 172, no. 10, pp. 6229-6238, 2004.

[4] H. S. Goodridge, F. A. Marshall, K. J. Else et al., "Immunomodulation via novel use of TLR4 by the filarial nematode phosphorylcholine-containing secreted product, ES-62," Journal of Immunology, vol. 174, no. 1, pp. 284-293, 2005.

[5] A. Loukas and P. Prociv, "Immune responses in hookworm infections," Clinical Microbiology Reviews, vol. 14, no. 4, pp. 689-703, 2001.

[6] A. L. Williamson, P. J. Brindley, D. P. Knox, P. J. Hotez, and A. Loukas, "Digestive proteases of blood-feeding nematodes," Trends in Parasitology, vol. 19, no. 9, pp. 417-423, 2003.

[7] S. M. Geiger, I. R. Caldas, B. E. Mc Glone et al., "Stage-specific immune responses in human Necator americanus infection," Parasite Immunology, vol. 29, no. 7, pp. 347-358, 2007. 
[8] N. Katz, A. Chaves, and J. Pellegrino, "A simple device for quantitative stool thick-smear technique in schistosomiasis mansoni," Revista do Instituto de Medicina Tropical de São Paulo, vol. 14, no. 6, pp. 397-400, 1972.

[9] A. Carr and D. I. Pritchard, "Antigen expression during development of the human hookworm, Necator americanus (Nematoda)," Parasite Immunology, vol. 9, no. 20, pp. 219234, 1987.

[10] L. R. Ash and T. C. Orihel, In Atlas of Human Parasitology, ASCP Press, Chicago, Ill, USA, 4th edition, 1997.

[11] J. M. Hawdon, S. Narasimhan, and P. J. Hotez, "Ancylostoma secreted protein 2: cloning and characterization of a second member of a family of nematode secreted proteins from Ancylostoma caninum," Molecular and Biochemical Parasitology, vol. 99, no. 2, pp. 149-165, 1999.

[12] S. M. Geiger, C. L. Massara, J. Bethony, P. T. Soboslay, O. S. Carvalho, and R. Corrêa-Oliveira, "Cellular responses and cytokine profiles in Ascaris lumbricoides and Trichuris trichiura infected patients," Parasite Immunology, vol. 24, no. 11-12, pp. 499-509, 2002.

[13] World Health Organization, Prevention and Control of Schistosomiasis and Soil-Transmitted Helminthiasis, WHO Technical Report Series, vol. 912, World Health Organization, Geneva, Switzerland, 2002.

[14] E. A. Ottesen, P. F. Weller, and L. Heck, "Specific cellular immune unresponsiveness in human filariasis," Immunology, vol. 33, no. 3, pp. 413-421, 1977.

[15] W. F. Piessens, P. B. McGreevy, P. W. Piessens et al., "Immune responses in human infections with Brugia malayi. Specific cellular unresponsiveness to filarial antigens," Journal of Clinical Investigation, vol. 65, no. 1, pp. 172-179, 1980.

[16] I. R. C. Viana, A. Sher, O. S. Carvalho et al., "Interferongamma production by peripheral blood mononuclear cells from residents of an area endemic for Schistosoma mansoni," Transactions of the Royal Society of Tropical Medicine and Hygiene, vol. 88, no. 4, pp. 466-470, 1994.

[17] C. L. King, A. Medhat, I. Malhotra et al., "Cytokine control of parasite-specific anergy in human urinary schistosomiasis. IL10 modulates lymphocyte reactivity," Journal of Immunology, vol. 156, no. 12, pp. 4715-4721, 1996.

[18] J. L. Grogan, P. G. Kremsner, A. M. Deelder, and M. Yazdanbakhsh, "Elevated proliferation and interleukin-4 release from CD4+ cells after chemotherapy in human Schistosoma heamatobium infection," European Journal of Immunology, vol. 26, no. 6, pp. 1365-1370, 1996.

[19] S. M. Geiger, C. L. Massara, J. Bethony, P. T. Soboslay, and R. Corrêa-Oliveira, "Cellular responses and cytokine production in post-treatment hookworm patients from an endemic area in Brazil," Clinical and Experimental Immunology, vol. 136, no. 2, pp. 334-340, 2004.

[20] A. Loukas, J. Croese, J. Opdebeeck, and P. Prociv, "Immunologic incrimination of Ancylostoma caninum as a human enteric pathogen," American Journal of Tropical Medicine and Hygiene, vol. 50, no. 1, pp. 69-77, 1994.

[21] P. Prociv and J. Croese, "Human enteric infection with Ancylostoma caninum: hookworms reappraised in the light of a 'new' zoonosis," Acta Tropica, vol. 62, no. 1, pp. 23-44, 1996.

[22] V. Wright and Q. Bickle, "Immune responses following experimental human hookworm infection," Clinical and Experimental Immunology, vol. 142, no. 2, pp. 398-403, 2005.

[23] S. Mendez, J. G. Valenzuela, W. Wu, and P. J. Hotez, "Host cytokine production, lymphoproliferation, and antibody responses during the course of Ancylostoma ceylanicum infection in the Golden Syrian Hamster," Infection and Immunity, vol. 73, no. 6, pp. 3402-3407, 2005.

[24] R. T. Fujiwara, S. M. Geiger, J. Bethony, and S. Mendez, "Comparative immunology of human and animal models of hookworm infection," Parasite Immunology, vol. 28, no. 7, pp. 285-293, 2006.

[25] G. C.-F. Hsieh, A. Loukas, A. M. Wahl et al., "A secreted protein from the human hookworm Necator americanusbinds selectively to NK cells and induces IFN- $\gamma$ production," Journal of Immunology, vol. 173, no. 4, pp. 2699-2704, 2004.

[26] H. J. McSorley and A. Loukas, "The immunology of human hookworm infections," Parasite Immunology, vol. 32, no. 8, pp. 549-559, 2010.

[27] J. Croese, M. J. Wood, W. Melrose, and R. Speare, "Allergy controls the population density of Necator americanus in the small Intestine," Gastroenterology, vol. 131, no. 2, pp. 402-409, 2006.

[28] J. M. Behnke, R. Rose, and J. Little, "Resistance of the hookworms Ancylostoma ceylanicum and Necator americanus to intestinal inflammatory responses induced by heterologous infection," International Journal for Parasitology, vol. 24, no. 1, pp. 91-101, 1994.

[29] B. M. Ogilvie, A. Bartlett, R. C. Godfrey, J. A. Turton, M. J. Worms, and R. A. Yeates, "Antibody responses in selfinfections with Necator americanus," Transactions of the Royal Society of Tropical Medicine and Hygiene, vol. 72, no. 1, pp. 6671, 1978.

[30] S. C. Chow, A. Brown, and D. I. Pritchard, "The human hookworm pathogen Necator americanus induces apoptosis in T lymphocytes," Parasite Immunology, vol. 22, no. 1, pp. 2129, 2000.

[31] C. Cuéllar, W. Wu, and S. Mendez, "The hookworm tissue inhibitor of metalloproteases (Ac-TMP-1) modifies dendritic cell function and induces generation of CD4 and CD8 suppressor T cells," PLoS neglected tropical diseases, vol. 3, no. 5, p. e439, 2009.

[32] S. Babu and T. B. Nutman, "Proinflammatory cytokines dominate the early immune response to filarial parasites," Journal of Immunology, vol. 171, no. 12, pp. 6723-6732, 2003.

[33] F. J. Culley, A. Brown, D. M. Conroy, I. Sabroe, D. I. Pritchard, and T. J. Williams, "Eotaxin is specifically cleaved by hookworm metalloproteases preventing its action in vitro and in vivo," Journal of Immunology, vol. 165, no. 11, pp. 64476453, 2000.

[34] J. Pesce, M. Kaviratne, T. R. Ramalingam et al., "The IL21 receptor augments Th2 effector function and alternative macrophage activation,” Journal of Clinical Investigation, vol. 116, no. 7, pp. 2044-2055, 2006.

[35] S. J. Jenkins, J. P. Hewitson, G. R. Jenkins, and A. P. Mountford, "Modulation of the host's immune response by schistosome larvae," Parasite Immunology, vol. 27, no. 10-11, pp. 385-393, 2005.

[36] M. Segura, Z. Su, C. Piccirillo, and M. M. Stevenson, "Impairment of dendritic cell function by excretory-secretory products: a potential mechanism for nematode-induced immunosuppression," European Journal of Immunology, vol. 37, no. 7, pp. 1887-1904, 2007.

[37] R. T. Fujiwara, G. G. L. Cançado, P. A. Freitas et al., "Necator americanus infection: a possible cause of altered dendritic cell differentiation and eosinophil profile in chronically infected individuals," PLoS Neglected Tropical Diseases, vol. 3, no. 3, article e399, 2009. 
[38] T. Kreider, R. M. Anthony, J. F. Urban, and W. C. Gause, "Alternatively activated macrophages in helminth infections," Current Opinion in Immunology, vol. 19, no. 4, pp. 448-453, 2007. 

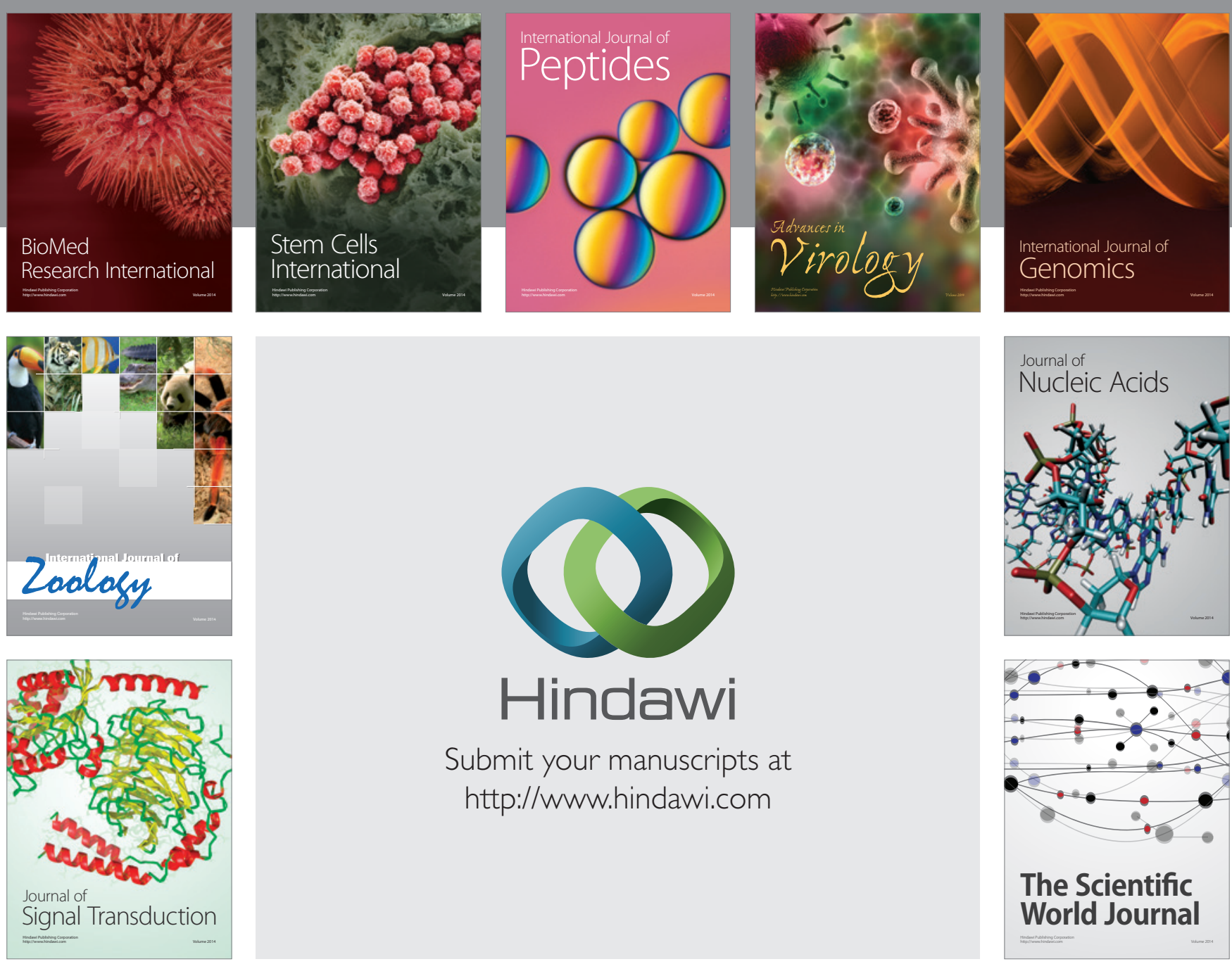

Submit your manuscripts at

http://www.hindawi.com
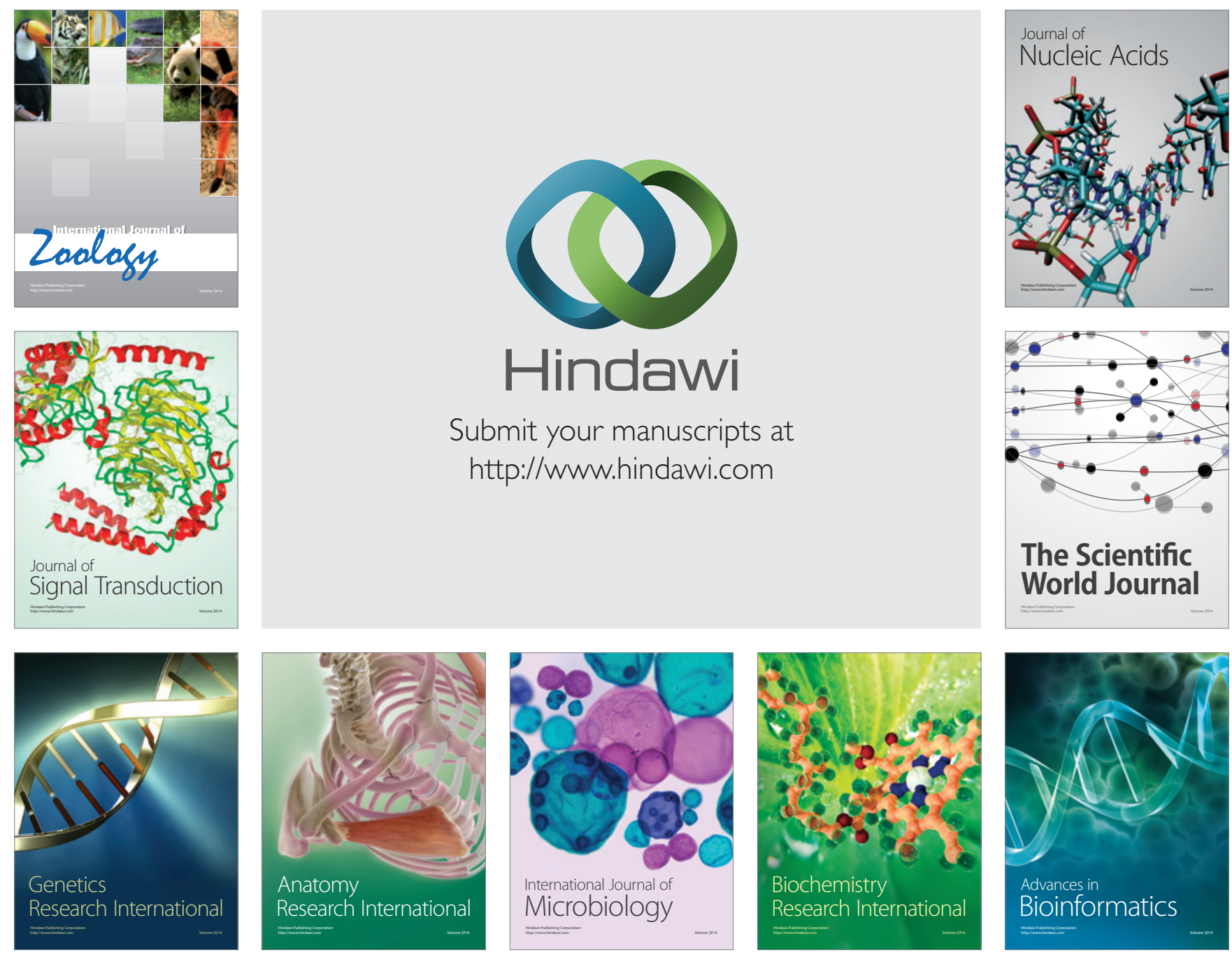

The Scientific World Journal
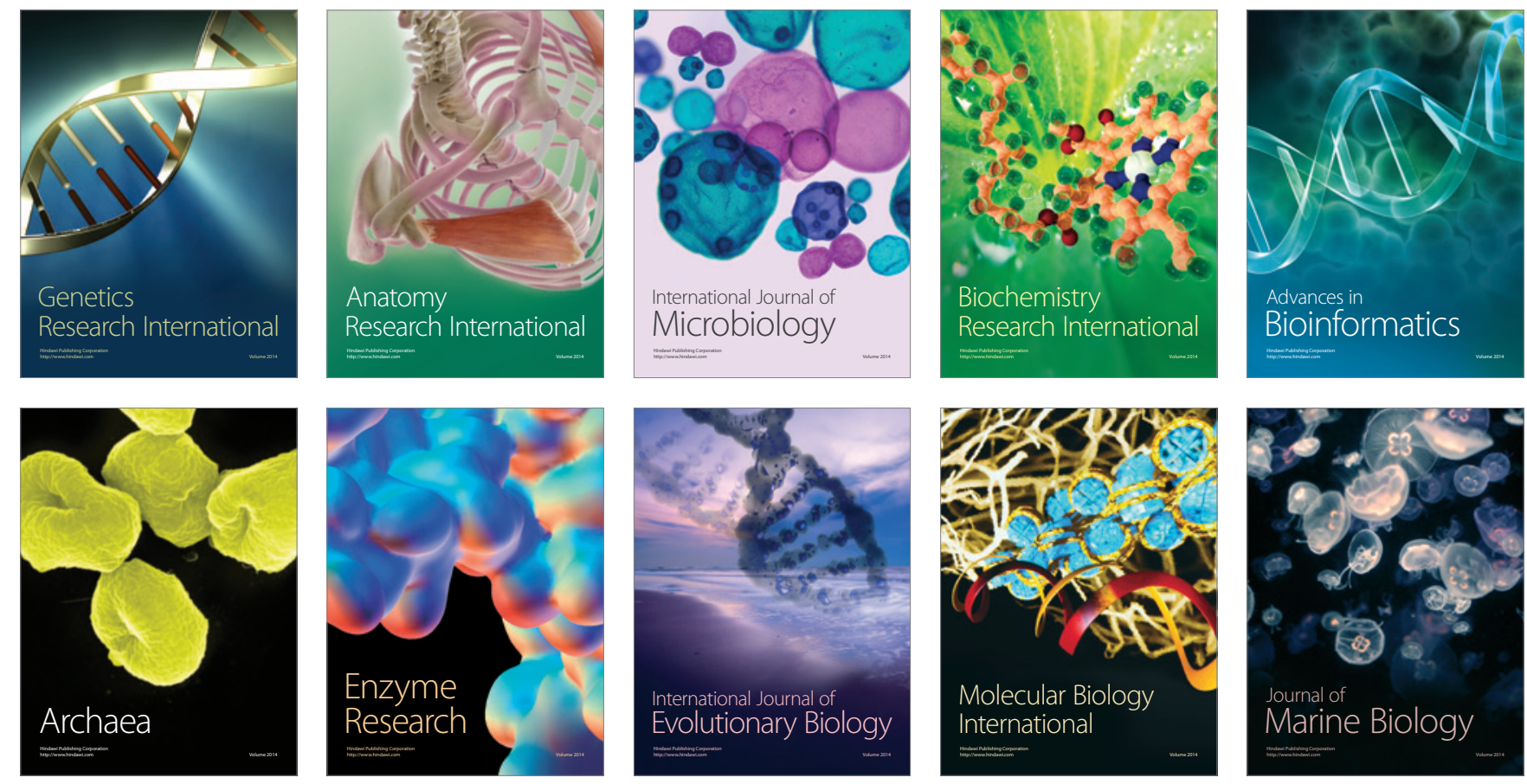